\title{
Cooperative dynamics in doped manganite films: Phonon anomalies in the ferromagnetic state
}

\author{
Ch. Hartinger, F. Mayr, and A. Loidl \\ EP V, Center for Electronic Correlations and Magnetism, University of Augsburg, 86135 Augsburg, Germany \\ T. Kopp \\ EP VI, Center for Electronic Correlations and Magnetism, University of Augsburg, 86135 Augsburg, Germany
}

(Received 26 March 2004; published 26 October 2004)

\begin{abstract}
We present optical measurements of phononic excitations in $\mathrm{La}_{2 / 3} \mathrm{Ca}_{1 / 3} \mathrm{MnO}_{3}$ (LCMO) and $\mathrm{La}_{2 / 3} \mathrm{Sr}_{1 / 3} \mathrm{MnO}_{3}$ (LSMO) thin films covering the full temperature range from the metallic ferromagnetic to the insulating paramagnetic phase. All eight phonons expected for the R $\overline{3 c}$ symmetry in LSMO and 17 out of the expected 25 phonons for the Pnma symmetry in LCMO have been determined. Close to the ferromagnetic-toparamagnetic transition both compounds reveal an anomalous behavior but with different characteristics. Anomalies in the phononic spectra are a manifestation of the coupling of lattice degrees of freedom (DOF) to electronic DOF. Specifically, the low-frequency external group proves to be an indicator for lattice modifications induced by electronic correlations. The enhanced electron-phonon coupling in LCMO is responsible for Fano-like interference effects of distinct phonon modes with electronic continuum excitations: we observe asymmetric phonon line shapes, mode splitting and spectral weight transfer between modes.
\end{abstract}

DOI: 10.1103/PhysRevB.70.134415

PACS number(s): 75.47.Lx, 72.80.-r, 78.20.-e, 75.40.Cx

\section{INTRODUCTION}

With the enormous progress of research on colossal magnetoresistance (CMR) manganites ${ }^{1}$ over the last decade it has been realized that besides charge and spin also lattice degrees of freedom ${ }^{2}$ play a significant role in the formation of the CMR and the metal-insulator transition (MIT). Special attention focused on the optimal doped manganites, characterized by the largest CMR effects - in particular, $\mathrm{La}_{2 / 3} \mathrm{Ca}_{1 / 3} \mathrm{MnO}_{3}$ (LCMO). As the temperature range in which the CMR effect and the MIT are observed is close to room temperature, these compounds have become potential candidates for technical applications.

The phonon excitation spectrum has been studied in detail for undoped $\mathrm{LaMnO}_{3}^{3,4}$ which is, below approximately $750 \mathrm{~K}$, in a Jahn-Teller (JT) distorted orthorhombic (Pnma) structure. A theoretical analysis of the complete set of phonon modes in the doped compounds is still missing. LCMO has an orthorhombic symmetry whereas the Sr-doped compound, $\mathrm{La}_{2 / 3} \mathrm{Sr}_{1 / 3} \mathrm{MnO}_{3}$ (LSMO), is rhombohedrally distorted. The infrared-active phonons of undoped rhombohedral $(\mathrm{R} 3 \mathrm{c}) \mathrm{LaMnO}_{3}$ were identified ${ }^{5}$ however these zerodoping investigations cannot make up for experiments at finite doping. In fact, the local $\mathrm{Mn}-\mathrm{O}$ geometry depends strongly on the size of the ions and doping concentration ${ }^{6-8}$ which accounts for the structural differences between LCMO and LSMO.

Experimental studies of two single crystals, undoped and with $\sim 8 \%$ Sr-doping, were performed by Paolone et al. ${ }^{9}$ They discussed the assignment of all infrared active phonon modes in $\mathrm{LaMnO}_{3}$ at low temperature. However, to the best of our knowledge, there are no reports on phonon modes in the metallic phase of LSMO. For bulk samples of LCMO, three sets of broad phonon bands are observed which reveal external-, bending- and stretching-type lattice vibrations, as classified in the cubic symmetry. ${ }^{10,11}$

Apart from providing a fingerprint of the lattice structure, phononic spectra can indicate the relevance of electronic cor- relations. The dominant effect of mobile electrons on the lattice vibrations is generically a suppression of phononic resonances due to screening. For metals, this may render the phonons unobservable in far-infrared (FIR) optical spectroscopy. However, since the polycrystalline samples are poor metals, and even more so the investigated thin films, phonon resonances are still clearly resolved even in the conducting low-temperature ferromagnetic phase.

In LCMO a significant shift of the stretching mode near the MIT was found ${ }^{10}$ and associated with a reduced screening through charge carriers close to the MIT. Although the observed shifts of phonons in LCMO are evidently related to electronic correlations since they come along with the MIT, it is not obvious that they are explained exclusively by a reduced screening. In particular, not all phonon modes display the shift and, furthermore, in LSMO we observe phononic shifts which are tied to the ferromagnetic-toparamagnetic (FM-PM) transition, not the MIT (see Sec. III C). In LCMO the FM-PM transition and the MIT coincide whereas in LSMO they are well separated. A comparative study of LCMO and LSMO should address and illuminate the fundamental question if the phononic excitations couple to spin degrees of freedom.

Since the phonon resonances are well pronounced in the optical spectroscopy measurements of the metallic manganite films they provide a unique opportunity to analyze the consequences of a coupling of lattice degrees of freedom (DOF) with spin and charge. Correlation of lattice DOF with charge DOF has to be in a weak (LSMO) to strong coupling range (LCMO) as, for example, the polaronic excitations in the mid-infrared (MIR) evidence a coupling in this range. ${ }^{12}$ Also neutron diffraction measurements for LCMO revealed an anomalous volume thermal expansion at the paramagnetic-to-ferromagnetic MIT, ${ }^{13-15}$ which has to originate from a sufficiently strong coupling. We will elaborate further on the consequences of a strong phonon-electron coupling for the phononic resonances in this paper and we will 
identify the characteristics in the FIR spectra signifying cooperative dynamics not only of lattice and electronic charge but also of lattice and spin degrees of freedom.

The paper is organized as follows: in Sec. II we deal with the experimental details, sample characterization and data analysis. In Sec. III A, we present reflectivity measurements of the infrared active phonons in thin films of LSMO and LCMO at temperatures ranging from the insulating paramagnetic (PM) to the metallic ferromagnetic (FM) phase. In Sec. III B we give a comprehensive analysis of phonons from the respective optical data. The spectra confirm the anticipated mode structure for the respective symmetry of the lattice and prove the high quality of the thin films. In Sec. III C we elaborate on the close relation between lattice and spin degrees of freedom by studying the temperature dependent shift of phonon modes across the ferromagnetic transition. In Sec. IV, we discuss Fano-like modifications of the line shape of distinct phonon modes in LCMO which testify a significant coupling of the lattice excitations to the electronic continuum in the Ca-doped manganite films. The interference of nearly degenerate modes with electronic excitations may result in spectral weight transfer and level repulsion which we discuss within a phenomenological approach for coupled phononic and electronic modes. A summary of our findings and conclusions are presented in the last section, Sec. V.

\section{EXPERIMENTAL DETAILS AND SAMPLE CHARACTERIZATION}

The films for this investigation have been prepared using a standard pulsed laser deposition technique. ${ }^{16}$ LCMO was grown to a thickness of $200 \mathrm{~nm}$ onto $\mathrm{NdGaO}_{3}$ single crystalline substrates. LSMO \#1 with a thickness of $d$ $=300 \mathrm{~nm}$ and LSMO $\# 2$ with $d=400 \mathrm{~nm}$ were deposited on $\left(\mathrm{LaAlO}_{3}\right)_{0.3}\left(\mathrm{Sr}_{2} \mathrm{AlTaO}_{5}\right)_{0.7}$. The x-ray diffraction of LCMO revealed an orthorhombic structure with a preferred growth along the [110] axis while LSMO was confirmed to be in a rhombohedral structure with preferred growth along the [100] axis.

The infrared reflectivities of the sample and the pure substrate were measured using a combination of Fourier transform spectrometers, Bruker IFS $113 \mathrm{v}$ and IFS $66 \mathrm{v} / \mathrm{S}$, to cover the frequency range from 50 to $40000 \mathrm{~cm}^{-1}$. Temperature dependent measurements from 6 to $295 \mathrm{~K}$ were performed with a He-cryostat. Higher temperatures were measured in a homemade oven in which the sample was exposed to a continuous flow of heated nitrogen gas. To obtain the optical conductivity $\sigma$ a Kramers-Kronig (KK) analysis was performed which included the results from submillimeter spectroscopy between 5 and $30 \mathrm{~cm}^{-1}$. Applying the Fresnel optical formulas for the complex reflectance coefficient of the substrate-film system, the optical conductivity for the films was calculated. ${ }^{17}$

Magnetization measurements were carried out between 2 and $400 \mathrm{~K}$ by using a commercial Quantum Design SQUID magnetometer. The dc resistance measurements were performed by a standard four-probe method. The temperature dependence of the ac resistancee was done in a MachZehnder interferometer arrangement, which allows the mea-
TABLE I. Metal-insulator $\left(T_{\mathrm{MIT}}\right)$, magnetic $\left(T_{C}^{\chi}\right)$ and electric $\left(T_{C}^{\rho}\right)$ transition temperatures of the LCMO and LSMO films.

\begin{tabular}{lcccl}
\hline \hline & $T_{\text {MIT }}(\mathrm{K})$ & $T_{C}^{\rho}(\mathrm{K})$ & $T_{C}^{\chi}(\mathrm{K})$ & Sample \\
\hline LCMO $(d=200 \mathrm{~nm})$ & 245 & 243 & 242 & LCMO \\
LSMO $(d=300 \mathrm{~nm})$ & 338 & 287 & 300 & LSMO \#1 \\
LSMO $(d=400 \mathrm{~nm})$ & 401 & 328 & 345 & LSMO \#2 \\
\hline \hline
\end{tabular}

surements of both transmission and phase shift. Applying the Fresnel formulas, the ac resistance was determined directly without any approximations.

Frequently, manganite films exhibit FM transition temperatures $T_{C}$ lower than those reported for the bulk materials with the same nominal composition. The differences can be attributed to grain boundaries, strain, microstructure depending on the details of the growth process and on the lattice parameters of the substrate. ${ }^{18,19}$ They all result in a variation of the strength of the lattice distortion, which determines the structure and, accordingly, the electrical and magnetic behavior. It is well known, that differences between bulk samples and thin films can occur, depending on the quality of the films. However, we observe that the overall optical characteristics of the films are in good agreement with bulk properties.

To probe the electrical and magnetic properties of the different films we determined the characteristic transition temperatures. We define $T_{C}^{\chi}$ as the magnetic Curie temperature, $T_{C}^{\rho}$ as the inflection point ${ }^{20}$ and $T_{\mathrm{MIT}}$ is taken from the maximum in the dc and ac resistivity curve. We summarize the results of the transition temperatures for both compounds in Table I. Since $T_{C}^{\chi}$ and $T_{C}^{\rho}$ are relatively similar, we will use the notation $T_{C}$ for either one if not stated otherwise. To identify the MIT and FM-PM transition of the thin film samples, we display magnetization and dc as well as ac resistivity in Fig. 1. As expected, $T_{C}$ nearly coincides with $T_{\mathrm{MIT}}$ for LCMO.

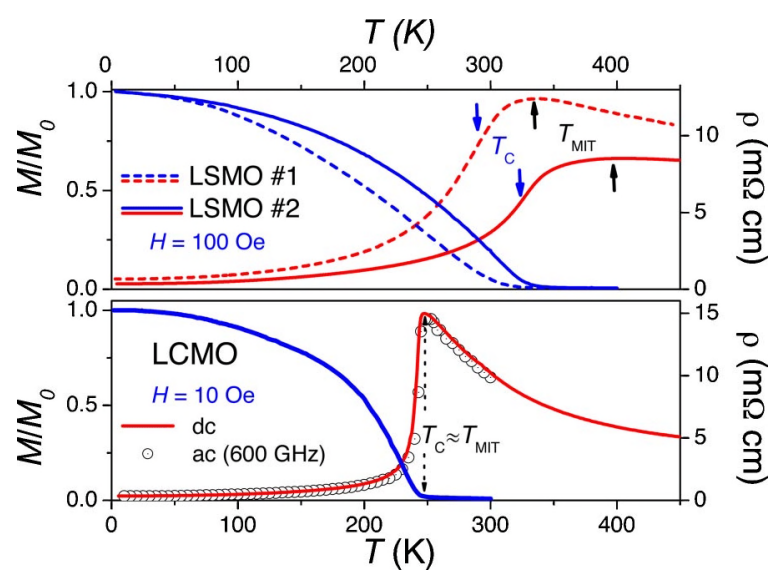

FIG. 1. Upper panel (LSMO): Temperature dependence of the magnetization at $H=100 \mathrm{Oe}$ (left scale) and DC-resistivity (right scale). Lower panel (LCMO): Magnetization at $H=10$ Oe (left scale); DC- and AC-resistivity at $\nu=600 \mathrm{GHz}$ (right scale). 


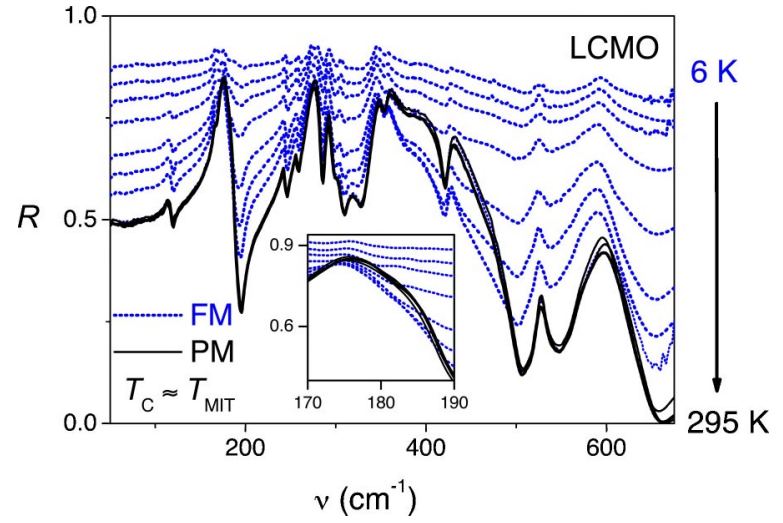

FIG. 2. Temperature dependence of the reflectivity $R$ of LCMO. The dotted lines represent temperatures in the FM phase, the solid lines in the PM phase. Above the crossover the intensity of $R$ is approximately temperature-independent. The anomalies of some phonons at $T_{C}$ evidence the significant role of electronic DOF in the modification of the lattice and its vibrations at the FM-PM MIT. Most pronounced is the enhancement of the reflectivity near the bending modes but an increment of $R$ is also observable for the higher-frequency external modes; cf. the inset.

\section{EXPERIMENTAL RESULTS AND DISCUSSION}

\section{A. Temperature dependence of the reflectivity}

The frequency-dependent reflectivity of LCMO is presented in Fig. 2 for temperatures in the metallic FM (dotted lines) and in the insulating PM (solid lines) phase. Above the MIT $(245 \mathrm{~K})$ the shape and intensity of $R$ is temperature independent. With the temperature increasing from $6 \mathrm{~K}$ to $295 \mathrm{~K}$, the weight of several phonon modes gradually increases and a typical insulating behavior with an energy gap of about $680 \mathrm{~cm}^{-1}$ develops. Variations in position and intensity of some characteristic phonon modes are apparent.

The overall distinction between the reflectivity spectra of LCMO and LSMO is striking (compare Figs. 2 and 3). The less complex structure of the phonon spectrum of LSMO, see Fig. 3, is evidence for the higher symmetry of the unit cell. Above $400 \mathrm{~K}$ the spectra hardly change. The reflectivity in LSMO \#1 is slightly lower than in LSMO \#2, otherwise the spectra are essentially identical. The lower reflectivity is related to the reduced mobility of the charge carriers which results from stronger strain and boundary effects in the thinner film. This is consistent with the observation that $T_{C}$ and $T_{\text {MIT }}$ are smaller for LSMO \#1 (Fig. 1).

In addition, the LCMO spectra display phonon anomalies at $T_{C}$, a feature which is entirely missing in LSMO. X-ray and neutron powder diffraction studies demonstrate an anomalous volume thermal expansion for the Ca-doped compound at the MIT. ${ }^{13-15}$ Dai et al. ${ }^{15}$ show that this anomaly originates from variations of the $\mathrm{Mn}$ and $\mathrm{O}$ positions. The modified $\mathrm{Mn}-\mathrm{O}$ bond lengths and bond angles are expected to cause anomalies of those phonon modes which are particularly sensitive to variations of the $\mathrm{Mn}-\mathrm{O}$ geometry. Contrary to LCMO, LSMO exhibits no anomalies in the lattice parameters and in the unit cell volume around $T_{C} \cdot{ }^{21,22}$

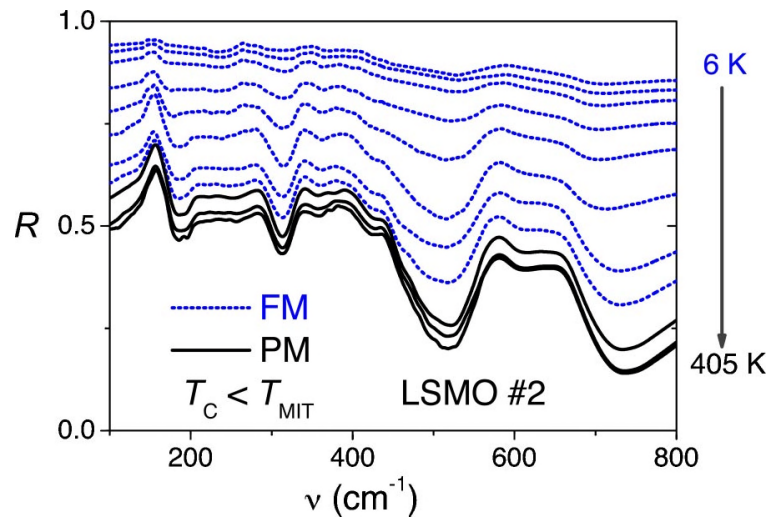

FIG. 3. Temperature dependence of the reflectivity $R$ of LSMO \#2. The dotted lines represent temperatures in the FM phase, the solid lines in the PM phase. With increasing temperature $R$ gradually decreases up to $T_{\mathrm{MIT}}=401 \mathrm{~K}$, which is above $T_{C}=345 \mathrm{~K}$.

\section{B. Phonon modes in LCMO and LSMO}

The different lattice structure of LSMO and LCMO has an impact on the optical conductivity, and on the number of observed phonon modes. Approximately, the phonon spectra of manganites can be separated into external $\left(\sim 185 \mathrm{~cm}^{-1}\right)$, bending $\left(\sim 350 \mathrm{~cm}^{-1}\right)$ and stretching $\left(\sim 550 \mathrm{~cm}^{-1}\right)$ modes with respect to cubic $(\mathrm{Pm} 3 \mathrm{~m})$ symmetry. Depending on ion size and doping concentration, these triply degenerate modes split into pairs of nondegenerate $(A)$ and doubly degenerate $(E)$ modes, and, moreover, they become broader and overlap. ${ }^{9,23}$ Furthermore, due to the larger unit cell additional modes emerge.

LSMO has a rhombohedral symmetry with space group $\mathrm{R} \overline{3 \mathrm{c}}$. The tilt of the octahedra results in a doubling of the cubic unit cell, and $8\left(5 E_{u}\right.$ and $\left.3 A_{2 u}\right)$ modes are IR active. LCMO has the Pnma structure with an orthorhombic unit cell containing four cubic units. Hence, 25 modes should be visible in the FIR spectra. In this section, we focus on spectra at high temperatures where the phonon peaks are better resolved.

The optical conductivity, $\sigma$, of LSMO \#2 at $250 \mathrm{~K}$ is obtained from the $\mathrm{KK}$ analysis of the reflectance data; see Fig. 4. We identify eight peaks of IR active transverse-optical (TO) phonon modes, corresponding to R $\overline{3 c}$-symmetry. A theoretical analysis with R $\overline{3 c}$-symmetry has been performed by Abrashev et al. ${ }^{5}$ for the phonon frequencies of $\mathrm{LaMnO}_{3}$. In Table II we present a comparison between the experimental values of the IR active phonon frequencies of LSMO \#2 at $T=250 \mathrm{~K}$ and the calculated frequencies. The calculated positions for the undoped compound can only be taken as a rough estimate for LSMO since the lattice constants and atomic positions are modified by Sr-doping. Also the assumption of equal $\mathrm{Mn}-\mathrm{O}$ bond lengths in the $\mathrm{MnO}_{6}$ octahedra $^{5}$ is not strictly valid for the doped compounds, since the JT effect breaks the symmetry dynamically and thereby affects the groups of bending and stretching modes. Finally, the unequal masses of the (La/Sr)-ions should influence the external mode. ${ }^{24,23}$ Several resonance frequencies 


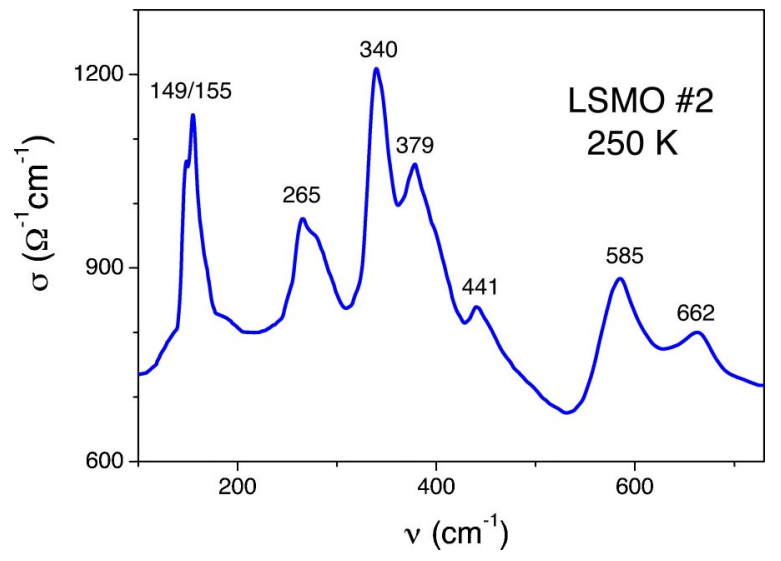

FIG. 4. Optical conductivity of the LSMO \#2 film at $250 \mathrm{~K}$. Those phonon modes which are expected in $\mathrm{R} \overline{\mathrm{c}} \mathrm{c}$ symmetry are labelled by numbers. They correspond to the phonon frequencies in units of $\mathrm{cm}^{-1}$.

for $\mathrm{La}_{0.7} \mathrm{Sr}_{0.3} \mathrm{MnO}_{3}$ are known from neutron scattering measurements, which are in good agreement with our data. ${ }^{25}$

In Fig. 5 the optical conductivity of LCMO at $250 \mathrm{~K}$ is plotted jointly with labels for the phonon eigenfrequencies. The latter result from a fit with noninteracting harmonic oscillators. From the series of observed sharp peaks below $\sim 600 \mathrm{~cm}^{-1}$ we can clearly identify 17 phonon modes. The theoretical studies for the Pnma symmetry performed by Fedorov et al. $^{3}$ and Smirnova ${ }^{4}$ were evaluated for the undoped case. Both sets of theoretical frequencies are remarkably different from the experimental data reported in detail by $\mathrm{Pa}-$ olone et al..$^{9}$ for $\mathrm{LaMnO}_{3}$.

In Table III the phonon frequencies for LCMO at $6 \mathrm{~K}$ and $250 \mathrm{~K}$ are listed and compared with published data on the infrared spectra of undoped $\mathrm{LaMnO}_{3} .{ }^{9}$ At $6 \mathrm{~K}$ we can identify 15 and at $250 \mathrm{~K} 17$ phonon modes. With increasing doping concentration weak phonon modes become broader and disappear in the background. Therefore not all 25 infrared active modes can be resolved. Remarkably, no phonon frequency larger than $600 \mathrm{~cm}^{-1}$ has been observed in LCMO. The deviations of the resonance frequencies in pure $\mathrm{LaMnO}_{3}$ from those in Ca-doped films are a consequence of a less distorted structure but enhanced disorder.

TABLE II. Correspondence between calculated (Ref. 5) and measured phonon modes for the space group $\mathrm{R} \overline{\mathrm{3}} \mathrm{c}$. The measured frequencies (in $\mathrm{cm}^{-1}$ ) refer to LSMO \#2 at $T=250 \mathrm{~K}$, and the values in parentheses to neutron scattering (Ref. 25) with a $\mathrm{La}_{0.7} \mathrm{Sr}_{0.3} \mathrm{MnO}_{3}$ sample.

\begin{tabular}{ccccc}
\hline \hline \multicolumn{2}{c}{ Calculated } & & \multicolumn{2}{c}{ Measured } \\
$A_{2 u}$ & $E_{u}$ & Assignment & $A_{2 u}$ & $E_{u}$ \\
\hline & 317 & Vibration (Mn) & 379 \\
162 & 180 & External & 149 & 155 \\
310 & 357 & Bending & $340(336)$ & $441(424)$ \\
641 & 642 & Stretching & $585(576)$ & 662 \\
& 240 & Torsional & & 265 \\
\hline \hline
\end{tabular}

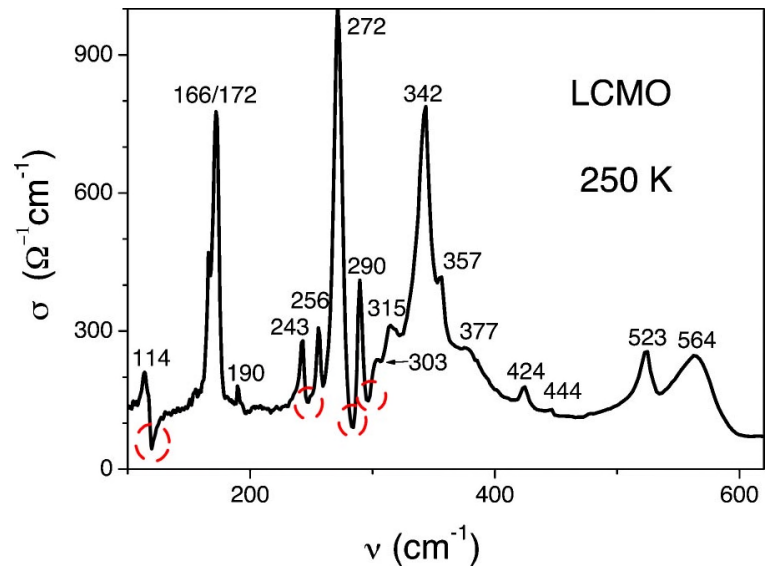

FIG. 5. Optical conductivity of the LCMO film at $250 \mathrm{~K}$. The numbers correspond to the phonon frequencies. The circles mark anomalies related to a strong electron-phonon coupling (see Sec. IV).

\section{Phononic resonances across the FM-PM transition}

The spectral resolution of phononic excitations in the films is superior to that in bulk LCMO samples and we can

TABLE III. A comparison of the phonon resonance frequencies (in $\mathrm{cm}^{-1}$ ) at $250 \mathrm{~K}$ and $6 \mathrm{~K}$ for the LCMO film with results by Paolone et al. (Ref. 9) for pure $\mathrm{LaMnO}_{3}$ at $300 \mathrm{~K}$ (P $300 \mathrm{~K}$ ) and at $10 \mathrm{~K}$ (P $10 \mathrm{~K})$.

\begin{tabular}{|c|c|c|c|c|}
\hline Osc. Nr. & P $10 \mathrm{~K}$ & $6 \mathrm{~K}$ & P $300 \mathrm{~K}$ & $250 \mathrm{~K}$ \\
\hline 1 & 116 & & 114 & 114 \\
\hline 2 & 119.5 & & & \\
\hline 3 & 162 & 166 & & 166 \\
\hline 4 & 172 & 175 & 172 & 172 \\
\hline 5 & 183.5 & 187 & 181.5 & 190 \\
\hline 6 & 201 & & 199 & \\
\hline 7 & 207 & & & \\
\hline 8 & 245 & 244 & 245 & 243 \\
\hline 9 & 249 & & & \\
\hline 10 & 268 & 259 & & 256 \\
\hline 11 & 275 & 273 & 275 & 272 \\
\hline 12 & 280 & 290 & 280 & 290 \\
\hline 13 & 287 & 305 & 287 & 303 \\
\hline 14 & 318 & 315 & 315 & 315 \\
\hline 15 & 340 & 344 & & 342 \\
\hline 16 & 352 & 355 & 350 & 357 \\
\hline 17 & 360 & 385 & 362 & 377 \\
\hline 18 & 400 & & 400 & \\
\hline 19 & 426 & 426 & 430 & 424 \\
\hline 20 & 434 & & & 444 \\
\hline 21 & 449 & & 450 & \\
\hline 22 & 478 & & 475 & \\
\hline 23 & 512 & 523 & 512 & 523 \\
\hline 24 & 561 & 589 & 563 & 589 \\
\hline 25 & 640 & & 640 & \\
\hline
\end{tabular}




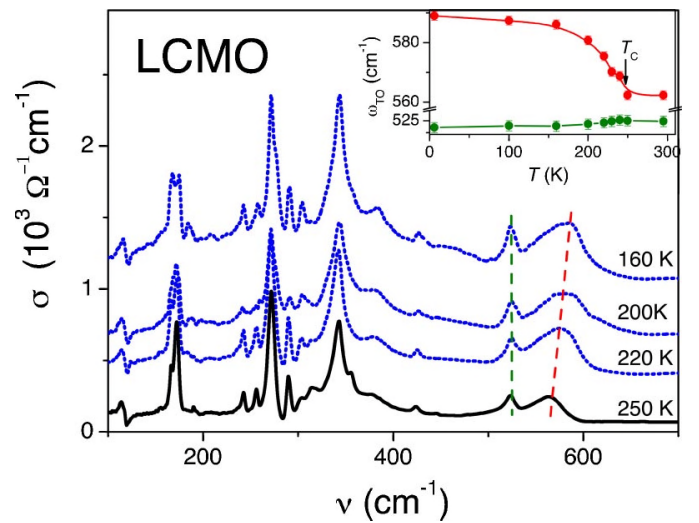

FIG. 6. Optical conductivity spectra of LCMO at various temperatures; dotted lines are spectra in the FM phase, solid lines in the PM phase. The dashed lines are to guide the eyes for the shift of the eigenfrequencies of the stretching modes. The higher mode changes significantly with temperature. The inset represents the eigenfrequency shift of both stretching modes versus temperature.

resolve even both stretching modes in the Pnma symmetry. Only the high-frequency stretching mode (see the inset of Fig. 6) exhibits a significant shift to lower energy with increasing temperature. This observation is to be related to the previously reported IR spectra of a polycrystalline LCMO sample by Kim et al. ${ }^{10}$ The authors observed only three broad modes of which the stretching mode displays a notable frequency shift around $T_{C}$. This shift on approaching the MIT was attributed to a reduction of screening through mobile charge carriers ${ }^{26}$ and, thereby, the authors depreciated a magneto-elastic effect. One argument against this interpretation is the observation that only one mode of the stretching group would be "sizeably less screened" 27 and we will see in Fig. 7 for the external modes that again only one mode shifts appreciably close to the MIT.

Some representative spectra of the phonon modes are seen in Fig. 6. Remarkably, at low temperatures a sharply resolved phononic structure remains which indicates that screening effects by mobile charge carriers play only a minor role. Consequently, we propose that the frequency shift of the high-frequency stretching mode (see the inset of Fig. 6) has a different origin. In this regard, the importance of spin-lattice correlations has been pointed out by Podobedov et al. ${ }^{28}$ who observed similar temperature effects of the phonon modes through polarized Raman spectra in an undoped sample (i.e., metallic screening is absent). ${ }^{29}$ Their assignment of the shift to spin-lattice correlations is in agreement with x-ray absorption fine-structure measurements for $\mathrm{La}_{1-x} \mathrm{Ca}_{x} \mathrm{MnO}_{3}$ by Booth et al. ${ }^{30}$ In the following we will further elucidate the correlation between the anomalous shift and the magnetization.

An intriguing example of a mode which exhibits the addressed anomaly in LCMO is the external mode (see the reflectivity in the inset of Fig. 2 and the optical conductivity in Fig. 7, left panel). The lowering of the symmetry from purely cubic to orthorhombic or rhombohedral implies a diversification of the three main phonon modes (external, bending and stretching) with the result that also the external mode depends on the Mn-O geometry. ${ }^{4}$
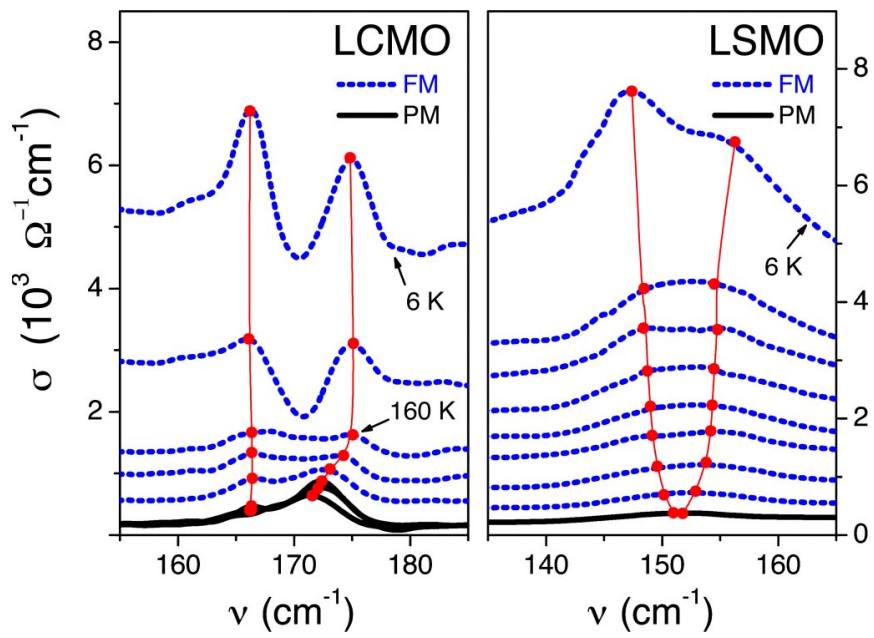

FIG. 7. A comparison of the external modes for LCMO (left) and LSMO (right); dotted lines are spectra in the FM phase, solid lines in the PM phase. For LCMO the shift of the high-frequency mode near MIT is clearly visible, while for LSMO the external modes become degenerate at about $T_{C}$. Temperatures for LCMO in K, from top to bottom: $6,100,160,200,220,240,250,295$, and for LSMO: 6, 100, 125, 160, 180, 200, 250, 295, 345.

As a consequence of the anharmonicity of the lattice an increase of the phonon resonance frequencies with decreasing temperature is expected. ${ }^{31}$ However, the considered phonon modes show an irregular temperature dependence (see Fig. 7 for the external modes, and the inset of Fig. 6 for the stretching modes). The shifts are controlled by the phase transition at $245 \mathrm{~K}$. In the left panel of Fig. 7 the positions of both external modes of LCMO are displayed for various temperatures. The lower mode exhibits only slight variations whereas the higher mode shows a clear anomaly which sets in at about $160 \mathrm{~K}$.

The observation that the magnetization (the solid line in Fig. 8) scales with the frequency shift of the higher external mode (solid circles) below $T_{C}$, suggests a correlation between spin polarization and this external mode. In comparison, the frequency shift of the high-energy stretching mode in the inset of Fig. 6 is seven times larger. As expected, the external mode is only slightly affected by the local, correlation-induced distortion of the $\mathrm{Mn}-\mathrm{O}$ octaedra. We point out that a link between magnetic correlations and phononic frequency shifts was realized before ${ }^{32}$ and it was applied successfully to explain the behavior of phonon modes in a ferromagnetic spinel. ${ }^{33}$

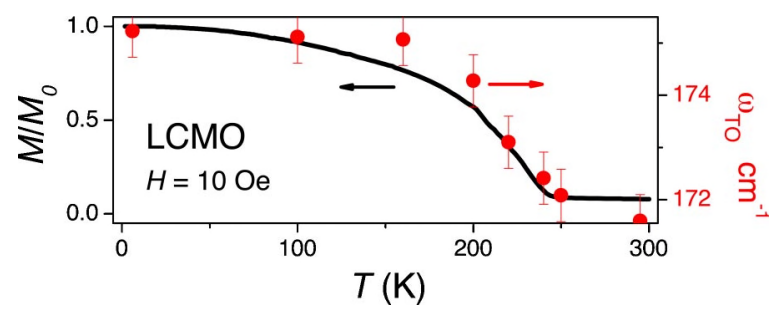

FIG. 8. Eigenfrequency of the high-energy external mode at various temperatures for LCMO (right scale). The solid line is the normalized magnetization (left scale). 


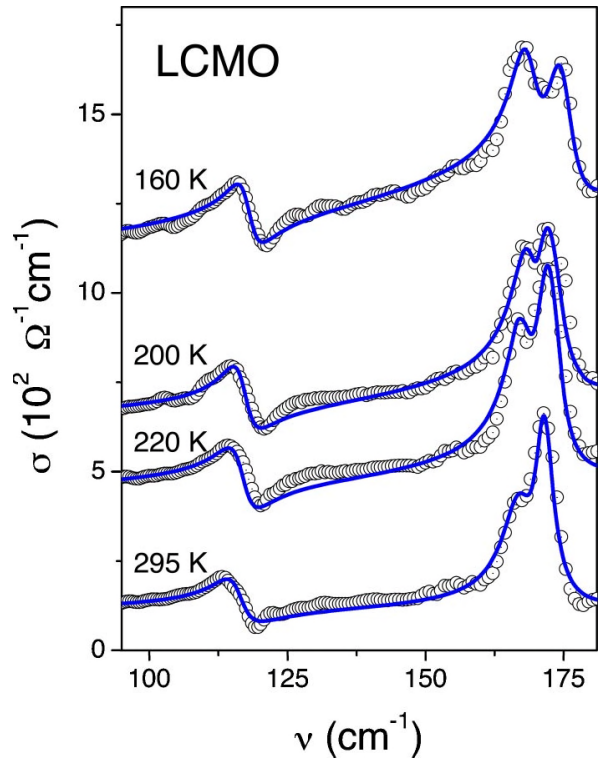

FIG. 9. Fano lineshape of the phonon mode at about $114 \mathrm{~cm}^{-1}$ and interference of the nearly degenerate external modes at about $170 \mathrm{~cm}^{-1}$ in LCMO. Fit with Eqs. (1)-(9) (line) to experimental data (open circles) at various temperatures.

The right panel of Fig. 7 presents the two external modes of LSMO. While the low-energy mode shows a softening with decreasing $T$, the higher mode displays the previously discussed hardening. Overall, the modes approach each other, and at $T_{C}$ hardly two modes are resolvable. ${ }^{34}$ We infer from this observation that the temperature scale of the mode evolution is in fact the magnetic temperature scale $T_{C}$.

Contrary to their behavior in LSMO, the two external modes in LCMO do not become degenerate when $T_{C}$ is approached. However in the next section, we will understand that electron-phonon coupling is much stronger in LCMO with consequences for the mode structure. In fact, the interference of nearby modes is pronounced in LCMO (see Fig. 9 ). We will discuss this observation within a phenomenological approach although, without a detailed microscopic analysis, it is not feasible to fix the position and weight of the considered phononic resonances conclusively. The formation of the different phononic resonances is strongly influenced by the details of electronic correlations and their coupling to the phonons. Nevertheless, we will see that for some of the modes, which strongly interact with the electronic correlations, a considerable amount of their weight may have its origin in a transfer of spectral weight from the electronic degrees of freedom (cf. the "bare" mode-amplitude $A_{2}$ in Table $\mathrm{V}$ with the resulting amplitude in Fig. 9 for the lower external mode at about $170 \mathrm{~cm}^{-1}$ ). It is conceivable that differences in the phononic mode structure of LSMO and LCMO - the shape and especially the relative weights of the phononic resonances - are, to a large extent, controlled by electron-phonon coupling.

\section{FANO LINESHAPES IN LCMO}

The lineshape of the phonon spectrum evidences the strength of the electron-lattice correlations as the coupling of lattice vibrations to excitations of the electronic system may lead either to destructive or constructive interference. The Lorentzian resonant shape of the phonon mode is thereby deformed into a resonance anti-resonance (peak-dip) structure if, for example, the phase of the electronic continuum excitations is nearly constant in the frequency range where the phononic excitation experiences a phase shift by $\pi$. The detailed form of the resonant structure then depends on the coupling strengths and the amplitudes of the considered excitations. A beautifully balanced peak-dip structure is observed for the lowest external mode at about $114 \mathrm{~cm}^{-1}$ in LCMO (see Fig. 9). Such an asymmetric Fano lineshape ${ }^{35,36}$ indicates a sizeable interaction between lattice vibrations and the electronic continuum. The asymmetry is also seen for other phonon modes (marked by open circles in Fig. 5). We observe these Fano lineshapes in LCMO, not in LSMO, where the electron-lattice coupling has to be considerably weaker.

Since a microscopic calculation of the resonance shape in these systems with charge, spin, orbital and lattice degrees of freedom is not yet feasible we discuss the asymmetry and coupling-dependent position and weight of the external modes within a phenomenological approach. It accounts for the phase relation between various oscillator modes which effectively model phonons and electronic continua. The coupling of these modes not only leads to Fano-like interference effects but also to mode splitting and the transfer of spectral weight. The parameters which enter the model (amplitudes, eigenfrequencies, relaxation rates and couplings) will be denoted as "bare parameters" and they will be determined from an optimal fit to the considered spectra. However it should be apprehended that they cannot be the bare parameters of a microscopic theory but rather contain residual interactions since the excitation spectrum cannot be fully decomposed into independent modes which couple in a scalar, linear way as assumed in the phenomenological modeling.

We analyze the present LCMO data by applying the phenomenological approach of Burlakov et al., ${ }^{37,38}$ taking into account several adjacent phonons which interact with electronic continua. The advantage of this approach is the explicit specification of and control over the electronic continua. In reference to our results in the mid-infrared, we take a small polaron (SP) resonance peaked between $1990 \mathrm{~cm}^{-1}$ at $160 \mathrm{~K}$ and $3300 \mathrm{~cm}^{-1}$ at room temperature ${ }^{12}$ Furthermore, we introduce an electronic oscillator in the far-infrared range, which has to be related to an incoherent contribution of orbital excitations ${ }^{39-41}$ in a unified treatment of all electronic and phononic degrees of freedom. As this cannot be the scope of the paper we identify these excitations phenomenologically with the spectral function of Eq. (8) (see below). This "electronic oscillator" contribution is peaked around $300 \mathrm{~cm}^{-1}$ in our optical measurements and it will be referred to as "incoherent contribution." We also include a Drude term which accounts for the small frequency side of the FIR spectrum but has only a marginal influence on the phonon modes.

For the evaluation of the optical conductivity $\sigma(\nu)$ we introduce a resonant interaction of the phonons with the electronic excitations: ${ }^{37,38}$ 


$$
\operatorname{Re} \sigma(\nu)=\varepsilon_{0} 2 \pi c \operatorname{Im}[\hat{A} \hat{G}(\nu) \hat{A}]
$$

where

$$
\hat{A}=\left(A_{1}, \ldots, A_{n}, A_{s p}, A_{e}, A_{d}\right)
$$

is a vector of $n$ matrix elements $\left(A_{j}\right)$ for optical dipole transitions of phonon modes, for the small polaron $\left(A_{s p}\right)$, for an incoherent $\left(A_{e}\right)$ and for a Drude contribution $\left(A_{d}\right)$. $\hat{G}$ is the Green's function matrix of the electron-phonon system. Thereby $A_{s p}$ is calculated by applying the $f$-sum rule to the SP component,

$$
A_{s p}=\sqrt{\frac{1}{\varepsilon_{0} 2 \pi^{2} c} \int_{0}^{\infty} d \nu \operatorname{Re} \sigma_{s p}(\nu)},
$$

where $\varepsilon_{0}$ is the vacuum permittivity and $c$ is the speed of light. The SP optical conductivity is ${ }^{42,43}$

$$
\operatorname{Re} \sigma_{s p}(\nu, T)=\sigma_{0}(T) \frac{\sinh \left(4 E_{b} \nu / \Delta^{2}\right)}{4 E_{b} \nu / \Delta^{2}} e^{-\nu^{2} / \Delta^{2}} .
$$

Here $\sigma_{0}(T)$ is the dc-conductivity, $E_{b}$ is the SP binding energy, $\Delta \equiv 2 \sqrt{2 E_{b} E_{\mathrm{vib}}}$ and $E_{\mathrm{vib}}$ is the characteristic vibrational energy which is the thermal energy $T$ in the high-temperature regime and $\nu_{\mathrm{ph}} / 2$ at low temperatures $\left(k_{B} T<\hbar 2 \pi c \nu_{\mathrm{ph}} ; \nu_{\mathrm{ph}}\right.$ is a phonon frequency). The dimension of $\nu$ and all other energy scales is $\mathrm{cm}^{-1}$.

We assume that not only the phonon modes approximately decouple but also the electronic modes, which generate the SP resonance, the Drude contribution and the resonance in the FIR range. The inverse Green's function matrix is in this phenomenological scheme:

$$
\hat{G}^{-1}=\left(\begin{array}{ccccccc}
G_{1}^{-1} & 0 & 0 & \ldots & g_{1} & k_{1} & 0 \\
0 & G_{2}^{-1} & 0 & \ldots & g_{2} & k_{2} & 0 \\
0 & 0 & G_{3}^{-1} & \ldots & g_{3} & k_{3} & 0 \\
\vdots & \vdots & \vdots & \ddots & \vdots & \vdots & \vdots \\
g_{1} & g_{2} & g_{3} & \ldots & G_{s p}^{-1} & 0 & 0 \\
k_{1} & k_{2} & k_{3} & \ldots & 0 & G_{e}^{-1} & 0 \\
0 & 0 & 0 & \ldots & 0 & 0 & G_{d}^{-1}
\end{array}\right),
$$

with the respective Green's functions of the phonon modes, the small polaron, incoherent and the Drude background:

$$
\begin{gathered}
G_{j}(\nu)=\frac{\nu}{\nu_{j}^{2}-\nu^{2}-i \nu \gamma_{j}}, \\
G_{s p}(\nu)=\frac{i \operatorname{Re} \sigma_{s p}(\nu)-\operatorname{Im} \sigma_{s p}(\nu)}{\varepsilon_{0} 2 \pi c A_{s p}^{2}}, \\
G_{e}(\nu)=\frac{\nu}{\nu_{e}^{2}-\nu^{2}-i \nu \gamma_{e}},
\end{gathered}
$$

TABLE IV. Parameters of the Fano-like fit for the $114 \mathrm{~cm}^{-1}$ phonon of LCMO at different $T$ using the approach of Eqs. (1)-(9).

\begin{tabular}{lcccc}
\hline \hline$T(\mathrm{~K})$ & 160 & 200 & 220 & 295 \\
\hline$A_{1}\left(\mathrm{~cm}^{-1}\right)$ & 150 & 148 & 143 & 140 \\
$\gamma_{1}\left(\mathrm{~cm}^{-1}\right)$ & 4.1 & 4.1 & 4.1 & 3.9 \\
$\nu_{1}\left(\mathrm{~cm}^{-1}\right)$ & 118.0 & 117.2 & 116.9 & 116.4 \\
$g_{1}\left(\mathrm{~cm}^{-1}\right)$ & -42 & -70 & -115 & -280 \\
$k_{1}\left(\mathrm{~cm}^{-1}\right)$ & -3 & -8 & -9 & -16 \\
$A_{s p}\left(\mathrm{~cm}^{-1}\right)$ & 16502 & 15251 & 15958 & 15495 \\
$\sigma_{0}\left(\Omega^{-1} \mathrm{~cm}^{-1}\right)$ & 890 & 460 & 350 & 108 \\
$E_{b}\left(\mathrm{~cm}^{-1}\right)$ & 1990 & 2000 & 2520 & 3300 \\
$\nu_{\mathrm{ph}}\left(\mathrm{cm}^{-1}\right)$ & 350 & 261 & 310 & 300 \\
$A_{d}\left(\mathrm{~cm}^{-1}\right)$ & 1350 & 1350 & 1350 & - \\
$\gamma_{d}\left(\mathrm{~cm}^{-1}\right)$ & 140 & 150 & 350 & - \\
$A_{e}\left(\mathrm{~cm}^{-1}\right)$ & 3500 & 3370 & 2800 & 1600 \\
$\gamma_{e}\left(\mathrm{~cm}^{-1}\right)$ & 370 & 340 & 280 & 250 \\
$\nu_{e}\left(\mathrm{~cm}^{-1}\right)$ & 276 & 320 & 340 & 345 \\
\hline \hline
\end{tabular}

$$
G_{d}(\nu)=-\frac{1}{\nu+i \gamma_{d}}
$$

$\gamma_{j}$ and $\nu_{j}$ are the width and the frequency of the $j$ th phonon mode, $\gamma_{e}$ and $\nu_{e}$ of the electronic oscillator, and $\gamma_{d}$ parametrizes the Drude contribution. The imaginary part of $\sigma_{s p}$ is obtained from a Kramers-Kronig relation. The strengths of the polaron-phonon $\left(g_{j}\right)$ and electron-phonon $\left(k_{j}\right)$ coupling parameters determine the asymmetric lineshape while the sign controls at which side of the phonon mode the dip will appear. For $g_{j}=k_{j}=0$ a Lorentzian shape is recovered for the $j$ th phonon. ${ }^{44}$ In relation (5) we set the coupling of the Drude-phonon coupling to zero as its effect on the resonant lineshapes is small for the considered temperature range below the MIT and we thereby avoid a larger number of fitting parameters. Moreover the Drude contribution is absent above the MIT but the lineshapes are still similar to those below the MIT, an observation which excludes a priori a dominant role of the Drude term. ${ }^{45,46}$

The fit procedure consists of the following three steps: First, the polaron parameters are fixed through the fit of the mid-infrared polaron resonance. ${ }^{12}$ Then the background in the FIR range is parametrized through $G_{e}$ and $G_{d}$. Finally, the frequencies and widths of the phonon resonances are determined, the coupling constants are extracted from fitting their respective shape, and the frequencies and widths are readjusted in order to gain the optimal fit.

In Fig. 9 we show experimental and theoretical data for the low-frequency phonon group measured at different temperatures. We now analyze in detail the most distinctive asymmetric mode in LCMO at about $114 \mathrm{~cm}^{-1}$ and include the two adjacent phonon modes for the interpretation. This group is well separated from other phonon excitations, which allows us to neglect the coupling to the remaining modes. In Table IV the interaction parameters on the basis of our approach are presented for the $114 \mathrm{~cm}^{-1}$ phonon for different temperatures $T$. 
TABLE V. Parameters of the $T$-dependent strong-coupling fit for the two external phonon modes of LCMO at about $170 \mathrm{~cm}^{-1}$, using the approach of Eqs. (1)-(9). Note that the frequencies in the table are the bare frequencies which differ from the interaction-dependent frequencies of the resonances in the optical conductivity, cf. Table III.

\begin{tabular}{lcccc}
\hline \hline$T(\mathrm{~K})$ & 160 & 200 & 220 & 295 \\
\hline$A_{2}\left(\mathrm{~cm}^{-1}\right)$ & 220 & 120 & 52 & 10 \\
$\gamma_{2}\left(\mathrm{~cm}^{-1}\right)$ & 5.0 & 4.3 & 3.6 & 3.2 \\
$\nu_{2}\left(\mathrm{~cm}^{-1}\right)$ & 169 & 169.5 & 169.8 & 171.1 \\
$g_{2}\left(\mathrm{~cm}^{-1}\right)$ & -7 & -3 & -2 & 0 \\
$k_{2}\left(\mathrm{~cm}^{-1}\right)$ & -16 & -28 & -47 & -65 \\
\hline$A_{3}\left(\mathrm{~cm}^{-1}\right)$ & 290 & 318 & 370 & 380 \\
$\gamma_{3}\left(\mathrm{~cm}^{-1}\right)$ & 5.0 & 4.7 & 4.5 & 4.2 \\
$\nu_{3}\left(\mathrm{~cm}^{-1}\right)$ & 175.0 & 172.8 & 172.5 & 171.5 \\
$g_{3}\left(\mathrm{~cm}^{-1}\right)$ & -9 & -4 & -2 & 0 \\
$k_{3}\left(\mathrm{~cm}^{-1}\right)$ & -12 & -14 & -22 & -28 \\
\hline \hline
\end{tabular}

All lineshapes of the first phonon mode in Fig. 9 exhibit a dip on the high-energy side, a consequence of the negative sign of the coupling parameters. The coupling constant is proportional to the effective mass. In manganites the carriers have hole character. ${ }^{47,48}$ The associated inverse sign of the effective mass may be responsible for a negative value of $g_{j}$ and $k_{j}$. It turns out that neither a coupling to the Drude nor to the incoherent contribution can induce the nearly perfect antisymmetric shape of the $114 \mathrm{~cm}^{-1}$ peak-dip structure. Only a sufficiently strong coupling to the polaronic excitation supports this antisymmetric Fano shape. The increase of coupling strength $g_{1}$ with temperature is significant. It results from the fact that the lineshape keeps its antisymmetric form up to the highest temperature $(295 \mathrm{~K})$ while the electronic background is reduced by a factor 8 from $160 \mathrm{~K}$ to $295 \mathrm{~K}$. Since the lineshape of the phonon is controlled by a product of the coupling constant and the strength of the polaronic background $\left(\sigma_{0}\right)$, the coupling constant has to increase correspondingly to keep the observed shape.

If one traces the phonon-continuum coupling reversely to lower temperatures (cf. Table IV) one might assume that the coupling extrapolates to zero. The strong reduction of these couplings with decreasing temperature is to be expected since the enhanced screening in the metallic phase at lower temperatures diminishes the value of these couplings sizably. For this reason, the antisymmetric lineshape should be less pronounced at lower temperatures. On the other hand, the spectral weight of the continuum increases with decreasing temperature as the material becomes more metallic (which, for example, reduces the binding energy of the polaron and therefore shifts its dominant spectral part to lower frequencies). With the increased weight of the continuum, the Fanolineshape has to be more pronounced. Obviously, the tendency towards a smaller coupling and the tendency towards increased continuum weight compete for the asymmetric Fano-lineshape and the seeming contradiction, that the lineshape changes little, is resolved. Certainly, it would be interesting to trace these tendencies down to the lowest tempera-

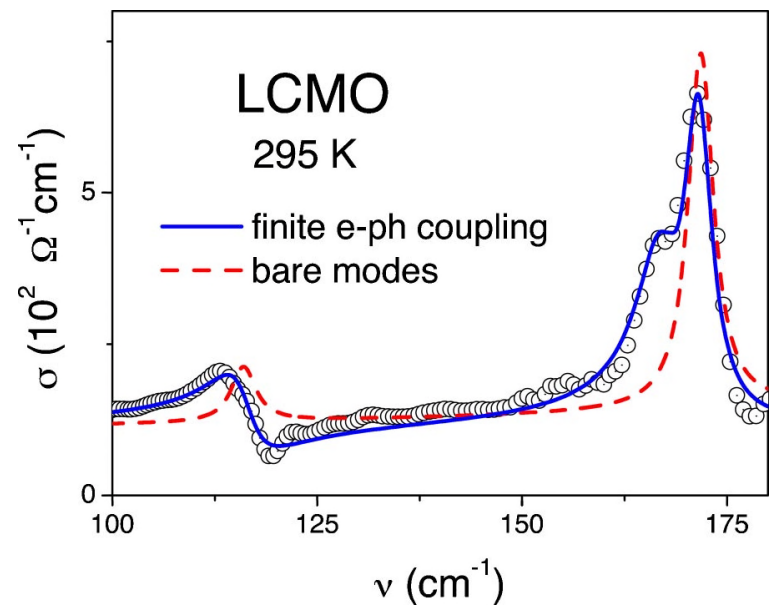

FIG. 10. Fits of the low frequency phonon group with and without electron-phonon (e-ph) coupling for data at room temperature.

tures (below the $160 \mathrm{~K}$ of Table IV). However the screening in the low-temperature metallic phase does not allow us to identify a well-resolved lineshape of the phonon modes in order to discuss the asymmetry at very low temperatures.

The lineshapes of the two modes at about $170 \mathrm{~cm}^{-1}$ do not deviate strongly from Lorentzians which is consistent with rather small couplings $g_{2}$ and $g_{3}$ (cf. Table V). However, in order to reproduce the "hump" in between the phonon at $114 \mathrm{~cm}^{-1}$ and the nearly degenerate external modes at about $170 \mathrm{~cm}^{-1}$, one needs a sufficiently strong electronphonon coupling $k_{2}$ to the electronic resonance at about $300 \mathrm{~cm}^{-1}$. These electron-phonon couplings, $k_{2}$ and $k_{3}$, affect the position and the spectral weight of the resonances at $\nu_{2}$ and $\nu_{3}$ considerably. The three coupled modes $\left(\nu_{2}, \nu_{3}, \nu_{e}\right)$ constitute a "triad" with a bonding (lowest), nonbonding (intermediate) and anti-bonding state. The bonding and antibonding modes always experience level repulsion so that the bare frequencies $\nu_{2}$ and $\nu_{3}$ can be both at $171 \mathrm{~cm}^{-1}$ (degenerate modes, cf. Table V) but the two resonances are still well separated at $295 \mathrm{~K}$ (at $167 \mathrm{~cm}^{-1}$ and at $171 \mathrm{~cm}^{-1}$; cf. Fig. 9 and Table III). ${ }^{49}$ The level repulsion is accompanied by a transfer of spectral weight: according to Table $\mathrm{V}$ and Fig. 9, the lowest mode of the triad takes nearly all its weight from the electronic mode at $295 \mathrm{~K}$. This observation may explain why the two-mode structure at $170 \mathrm{~cm}^{-1}$ in LCMO is still intense at or above the MIT whereas in LSMO the mode at about $170 \mathrm{~cm}^{-1}$ is weak (cf. Fig. 7, right panel). In Fig. 10 we display the "bare modes," with parameters of Tables IV and $\mathrm{V}$, jointly with the interaction-dominated mode spectrum in order to summarize visually the effects of the strong electron-coupling on the external mode group in LCMO.

In contrast to LCMO, the phonon spectra for LSMO exhibit no asymmetric shape and can be described by Lorentzian oscillators. In the latter compound the electron-phonon coupling is smaller, as is confirmed by shape, position and temperature dependence of the polaronic mid-infrared (MIR) resonance. $^{12}$

\section{CONCLUSIONS}

Manganite thin films provide an excellent opportunity to study the phononic excitations of LSMO and LCMO in their 
metallic phases. The films allow for a well-resolved, distinct phonon spectrum in infrared spectroscopy, even for temperatures well below the MIT. In LSMO we identified each of the eight infrared-active phonons expected for the R $\overline{3 c}$ symmetry and in LCMO we were able to resolve 17 out of 25 phonons expected for the Pnma-symmetry.

The phonon spectra reveal a number of anomalies which result from a finite electron-lattice coupling and which confirm the importance of cooperative effects of microscopic degrees of freedom when approaching the ferromagnetic-toparamagnetic and the metal-insulator transition.

First and most apparent, we observe that the FIR spectra change substantially up to the MIT (see Figs. 2 and 3) but then, above the MIT, temperature dependent modifications are negligible. This overall $T$-dependence of the spectra up to the MIT is related to a strongly reduced screening of the phononic excitations, due to localization or diffusive motion of electronic charges. However also a coupling of lattice and spin degrees of freedom is evidenced by the phononic excitation spectrum: temperature dependent shifts of several phonon modes, which vary on the same temperature scale as the magnetization, confirm the correlation of these degrees of freedom (see Figs. 6-8.

In LCMO the pronounced asymmetric line shapes, observed for several phonons (see Figs. 5 and 9), are a manifestation of the strong electron-phonon coupling. We analyzed the Fano lineshapes of the external group within a phenomenological approach. ${ }^{37,38}$ It assigns independent modes to the various spectral contributions of the electronic excitations and couples them linearly to the phononic oscillators. In FIR and MIR spectra of LCMO, three electronic continua are typically identified: a "continuum of incoherent electronic excitations" (cf. Refs. 39-41), observed as a background to the phonons in the FIR range, polaronic excitations in the MIR and possibly a rather insignificant Drude contribution on the low-frequency side of the FIR spectrum.

The lineshape of the lowest-frequency external mode, which is remarkably antisymmetric at temperatures from $160 \mathrm{~K}$ to $295 \mathrm{~K}$, can be reproduced for high temperatures only with a sufficiently strong coupling to the polaronic excitations. In contrast, the FIR continuum, which is parametrized by a strongly damped oscillator spectral function, is responsible for frequency shifts (level repulsion) and transfer of spectral weight from the electronic excitations into distinct phononic modes (see Fig. 10). In the presented scheme, we find that the mode splitting of the $170 \mathrm{~cm}^{-1}$ resonance and its considerable spectral weight at high temperature (above the MIT) is induced by the electronic continuum. This observation may explain the discrepancy with LSMO where these two external modes are degenerate at high temperature since LSMO is characterized by a weaker electronphonon coupling where Fano-like lineshapes are entirely missing. A microscopic evaluation is mandatory in order to assign unambiguously these anomalous characteristics of the phononic spectra to the interference with specific electronic excitations. The preliminary identification within the phenomenological approach has provided ample motivation for such an effort.

\section{ACKNOWLEDGMENTS}

The research was supported by BMBF (13N6917, 13N6918) and by DFG through the Sonderforschungsbereich SFB 484 (Augsburg).
${ }^{1}$ R. von Helmolt, J. Wecker, B. Holzapfel, L. Schultz, and K. Samwer, Phys. Rev. Lett. 71, 2331 (1993).

${ }^{2}$ A. J. Millis, P. B. Littlewood, and B. I. Shraiman, Phys. Rev. Lett. 74, 5144 (1995).

${ }^{3}$ I. Fedorov, J. Lorenzana, P. Dore, G. DeMarzi, P. Maselli, P. Calvani, S. W. Cheong, S. Koval, and R. Migoni, Phys. Rev. B 60, 11875 (1999).

${ }^{4}$ I. S. Smirnova, Physica B 262, 247 (1999).

${ }^{5}$ M. V. Abrashev, A. P. Litvinchuk, M. N. Iliev, R. L. Meng, V. N. Popov, V. G. Ivanov, R. A. Chakalov, and C. Thomsen, Phys. Rev. B 59, 4146 (1999).

${ }^{6}$ H. Y. Hwang, S-W. Cheong, P. G. Radaelli, M. Marezio, and B. Batlogg, Phys. Rev. Lett. 75, 914 (1995).

${ }^{7}$ J. B. Goodenough, Magnetism and the Chemical Bond (Interscience, New York, 1963).

${ }^{8}$ A. Urushibara, Y. Moritomo, T. Arima, A. Asamitsu, G. Kido, and Y. Tokura, Phys. Rev. B 51, 14103 (1995).

${ }^{9}$ A. Paolone, P. Roy, A. Pimenov, A. Loidl, O. K. Melnikov, and A. Y. Shapiro, Phys. Rev. B 61, 11255 (2000).

${ }^{10}$ K. H. Kim, J. Y. Gu, H. S. Choi, G. W. Park, and T. W. Noh, Phys. Rev. Lett. 77, 1877 (1996).

${ }^{11}$ A. V. Boris, N. N. Kovaleva, A. V. Bazhenov, P. J. M. van Bentum, Th. Rasing, S-W. Cheong, A. V. Samoilov, and N.-C. Yeh,
Phys. Rev. B 59, R697 (1999).

${ }^{12}$ Ch. Hartinger, F. Mayr, J. Deisenhofer, A. Loidl, and T. Kopp, Phys. Rev. B 69, 100403(R) (2004).

${ }^{13}$ P. G. Radaelli, D. E. Cox, M. Marezio, S-W. Cheong, P. E. Schiffer, and A. P. Ramirez, Phys. Rev. Lett. 75, 4488 (1995).

${ }^{14}$ J. M. De Teresa, M. R. Ibarra, J. Blasco, J. García, C. Marquina, P. A. Algarabel, Z. Arnold, K. Kamenev, C. Ritter, and R. von Helmolt, Phys. Rev. B 54, 1187 (1996).

${ }^{15}$ P. Dai, J. Zhang, H. A. Mook, S.-H. Liou, P. A. Dowben, and E. W. Plummer, Phys. Rev. B 54, R3694 (1996).

${ }^{16}$ D. Christey and G. Hubler, Pulsed Laser Deposition of Thin Films (Wiley, New York, 1994).

${ }^{17}$ O. S. Heavens, Optical Properties of Thin Solid Films (Dover, New York, 1991).

${ }^{18}$ D. C. Worledge, G. Snyder, M. R. Beasley, M. R. Geballe, R. Hiskes, and S. DiCarolis, J. Appl. Phys. 80, 5158 (1996).

${ }^{19}$ E. Gommert, H. Cerva, J. Wecker, and K. Samwer, J. Appl. Phys. 85, 5417 (1999).

${ }^{20}$ M. E. Fisher and J. S. Langer, Phys. Rev. Lett. 20, 665 (1968).

${ }^{21}$ M. C. Martin, G. Shirane, Y. Endoh, K. Hirota, Y. Moritomo, and Y. Tokura, Phys. Rev. B 53, 14285 (1996).

${ }^{22}$ A. Mellergård, R. L. McGreevy, and S. G. Eriksson, J. Phys.: Condens. Matter 12, 4975 (2000). 
${ }^{23}$ F. Mayr, Ph.D. thesis, Universität Augsburg, 2002.

${ }^{24}$ J. L. MacManus-Driscoll, G. T. Tan, W. Y. Hung, A. V. Berenov, J. A. Alonso, M. G. Blamire, F. Damay, and L. F. Cohen, J. Mater. Res. 15, 1167 (2000).

${ }^{25}$ W. Reichardt and M. Braden, Physica B 263, 416 (1999).

${ }^{26}$ U. Yu, B. I. Min, and J. D. Lee, Phys. Rev. B 61, 84 (2000).

${ }^{27}$ Moreover, a full evaluation of screening would have to include the diffusive nature of the charge transport which results in a retarded screening dynamics with a less prominent signature.

${ }^{28}$ V. B. Podobedov, A. Weber, D. B. Romero, J. P. Rice, and H. D. Drew, Phys. Rev. B 58, 43 (1998).

${ }^{29}$ Near the magnetic transition an irregular shift at $609 \mathrm{~cm}^{-1}$ was attributed to the spin-lattice interaction due to the observed variations of the lattice parameters and sublattice magnetization.

${ }^{30}$ C. H. Booth, F. Bridges, G. J. Snyder, and T. H. Geballe, Phys. Rev. B 54, R15 606 (1996).

${ }^{31}$ R. F. Wallis and A. A. Maradudin, Phys. Rev. 125, 1277 (1962).

${ }^{32}$ W. Baltensperger, J. Appl. Phys. 41, 1052 (1970).

${ }^{33}$ K. Wakamura, T. Arai, and K. Kudo, J. Phys. Soc. Jpn. 41, 130 (1976).

${ }^{34}$ The origin of the mode degeneracy in LSMO and its relation to the evolution of the spin degrees of freedom will be investigated in a subsequent publication (Hartinger et al., cond-mat/ 0404023).

${ }^{35}$ U. Fano, Phys. Rev. 124, 1866 (1961).

${ }^{36}$ M. Opel, R. Hackl, T. P. Devereaux, A. Virosztek, A. Zawadowski, A. Erb, E. Walker, H. Berger, and L. Forro, Phys. Rev. B 60, 9836 (1999).

${ }^{37}$ V. M. Burlakov, S. V. Shulga, J. Keller, and K. F. Renk, Physica C 190, 304 (1992).

${ }^{38}$ A. Zibold, M. Dürrler, A. Gaymann, H. P. Geserich, N. Nücker,
V. M. Burlakov, and P. Müller, Physica C 193, 171 (1992).

${ }^{39}$ P. Horsch, J. Jaklič, and F. Mack, Phys. Rev. B 59, 6217 (1999).

${ }^{40}$ H. Shiba, R. Shiina, and A. Takahashi, J. Phys. Soc. Jpn. 66, 941 (1997).

${ }^{41}$ S. Ishihara, M. Yamanaka, and N. Nagaosa, Phys. Rev. B 56, 686 (1997).

${ }^{42}$ A. V. Puchkov, T. Timusk, M. A. Karlow, S. L. Cooper, P. D. Han, and D. A. Payne, Phys. Rev. B 52, R9855 (1995).

${ }^{43}$ S. Yoon, H. L. Liu, G. Schollerer, S. L. Cooper, P. D. Han, D. A. Payne, S.-W. Cheong, and Z. Fisk, Phys. Rev. B 58, 2795 (1998).

${ }^{44}$ In the original Fano model a parameter $q$ determines the degree of the line asymmetry of the peak, which is inversely related to the strength of interaction. (Refs. 45 and 46).

${ }^{45}$ A. Damascelli, K. Schulte, D. van der Marel, and A. A. Menovsky, Phys. Rev. B 55, R4863 (1997).

${ }^{46}$ S. Lupi, M. Capizzi, P. Calvani, B. Ruzicka, P. Maselli, P. Dore, and A. Paolone, Phys. Rev. B 57, 1248 (1998).

${ }^{47}$ H. L. Ju, H.-C. Sohn, and K. M. Krishnan, Phys. Rev. Lett. 79, 3230 (1997).

${ }^{48}$ T. Saitoh, A. E. Bocquet, T. Mizokawa, H. Namatame, A. Fujimori, M. Abbate, Y. Takeda, and M. Takano, Phys. Rev. B 51, 13942 (1995).

${ }^{49}$ Although the scheme with a pure triad of bonding, nonbonding and anti-bonding modes is oversimplified, it visualizes sufficiently the expected modifications of mode positions and spectral weight with increasing coupling. Certainly, the higherenergy external mode at about $172 \mathrm{~cm}^{-1}$ has not a pure nonbonding character and it also experiences a small level shift and modifications of its spectral shape through its interaction with the other modes. 